\title{
Antibiotics susceptibility pattern and prevalence of isolated uropathogens in inpatient and out patients with lower urinary tract infections
}

\author{
Ranakishor Pelluri*, Paditham Monika, Harika Paritala, Chandrika Reddy Annapareddy, Bhuvaneswari Kotha, \\ Srujana Meenavilli, Sandhya Rani Angadi, Geethika Rayapati, Srinivasababu Puttagunta \\ Department of Pharmacy Practice, Vignan Pharmacy College, Guntur, Andhra Pradesh, India.
}

\begin{tabular}{l}
\hline ARTICLE INFO \\
\hline Received on: $19 / 06 / 2021$ \\
Accepted on: 09/10/2021 \\
Available Online: 05/01/2022 \\
\\
\hline Key words: \\
Lower UTI, uropathogens, \\
antibiotic susceptibility, in \\
and out patients, South India.
\end{tabular}

\begin{tabular}{l}
\hline ABSTRACT \\
Bacterial resistance was the consequence of empirical therapy and poor compliance, and its amelioration was difficult. \\
In both in-patients and out-patients, definitive therapy resulted in better outcomes for urinary tract infections (UTI). Our \\
objective was to identify the prevalence and to assess the susceptibility pattern of uropathogens toward antibiotics. It is \\
a prospective, cross-sectional study conducted during the period of November, 01,2019 to April, 28, 2020. A total of \\
200 UTI patient's antibiotic susceptibility pattern was analyzed of which, 105 were males and 95 were females. Among \\
them, 147 were in-patients and 53 were out-patients. The prevalence of UTI was high between the ages of 40 and 79 \\
years. Less than $20 \%$ susceptibility was noticed in $20-39$ years of age groups and less than $10 \%$ were observed in $80-99$ \\
years of age groups. The antibiotic susceptibility was found to be $35 \%-40 \%$ among the $40-59$ years of age group, of \\
them Ertapenem and Trimethoprime (TMP) \& Sulfamethoxazole (SMZ) only has $48.5 \%$ and $44.6 \%$ of susceptibility \\
and literally poor in $80-99$ years. Colistin and Amikacin showed higher susceptibility toward Escherichia coli and \\
Klebsiella pneumoniae in in-patients. The remaining antibiotics showed $40 \%-67 \%$ of susceptibility. TMP and SMZ, \\
Amoxicillin Clavulanic acid, Cefoperazone, and Amoxicillin plus Clavulanic acid had relatively low susceptibility \\
(16\%-27\%). Colistin, Amikacin, and Nitrofurantoin showed better susceptibility in in-patients and in out-patients. \\
TMP and SMZ, Amoxicillin Clavulanic acid, Cefoperazone, and Amoxicillin plus Clavulanic acid had relatively low \\
susceptibility. Escherichia coli was more prevalent Gram-negative bacteria isolated among the individuals and 57.8\% \\
of In-patients (IP) and $67.9 \%$ of out-patients (OP) with UTI were isolated with E. coli.
\end{tabular}

\section{INTRODUCTION}

Urinary tract infection (UTI) is a common health problem in both community and nosocomial settings, affecting both men and women equally. It is characterized by uropathogens colonization in the urinary tract, resulting in pyouria, dysuria, and urgency (Sarwar et al., 2013). Worldwide, approximately 6 million patients are visiting outpatient clinics each year and 3 million being treated in wards (Akortha et al., 2008; Medina and Castillo-Pino, 2019).

UTI episodes affect $50 \%-60 \%$ of women at some point in their lives (American College of Obstetricians and Gynecologists,

\section{"Corresponding Author}

Ranakishor Pelluri, Department of Pharmacy Practice, Vignan Pharmacy College, Guntur, India. E-mail: ranampharm@gmail.com
2008; Langer et al., 2021). The dreadful sequels of UTI are pyelonephritis, renal scarring, and renal failure (Foxman, 2010). Gram-negative bacteria such as Escherichia coli (E. coli), Proteus species, Pseudomonas aeruginosa (P. aeruginosa), Acinetobacter species, Klebsiella species, Enterobacter species, and Citrobacter species are commonly responsible for UTIs. Staphylococcus saprophyticus, Enterococcus species, and Coagulase-negative bacteria are Gram-positive bacteria, among them; Staphylococcus is traditional type of bacteria that cause infections (Momoh et al., 2011). Nearly $75 \%$ of uncomplicated UTIs are caused by E. coli (Flores et al., 2015).

Antibiotics are the mainstay of UTI treatment. Emerging antimicrobial resistance is the major issue in treating UTIs. However, with increasing reports of bacterial resistance to antibiotics, treating UTIs has become difficult (Sharef et al., 2015). Lack of hygiene, noncompliance with dose, duration of prescribed antibiotic(s), and unjustified prescription writing 
by quacks and doctors are all factors that have been shown to influence bacterial resistance. Furthermore, the easy availability of antibiotics as over-the-counter medication will contribute to the development of resistance (Kidwai et al., 2017). Several national and international surveillance systems have been developed to track the susceptibilities of clinically significant uropathogens causing infections (Hertz et al., 2016; Qiao et al., 2013). Another source of concern is the emergence of beta-lactamase producing Gram negative bacteria, such as E. coli and Klebsiella species, that are multidrug resistant not only to all Cephalosporin generations, but also to fluroquinolones and beta lactam inhibitor/lactamase inhibitor (Piperacillin/Tazobactam) combinations, leaving only Carbapenem as a treatment option (Mazzulli, 2012).

The purpose of this study was to determine the prevalence and antibiotic sensitivity pattern of the most common uropathogens isolated in both in-patients and out-patients with UTIs who visited the hospital. Several studies have been carried out in India; however, as a part of the continuous surveillance program, antibiotic resistance would need to be treated on a regular basis in order to change the guidelines accordingly.

\section{MATERIALS AND METHODS}

\section{Study design}

A single center, prospective and cross-sectional study conducted in patients with UTI was referred in the Central Laboratory of Ramesh Hospital, India, to analyze urine cultures from the age more than 18 years were collected in the inpatient and outpatient setting during the period of November, 01, 2019 to April, 28, 2020 (6 months). The mid-stream urine was collected in a suitable sterile container under hygienic conditions. The samples were incubated at $37^{\circ} \mathrm{C}$, and then it was investigated after 24-18 hours.

\section{Study population}

\section{Inclusion criteria}

The data were collected from the patients those who are diagnosed with UTI (both inpatients and out patients) with either sex of age more than 18 years and willing to give informed consent to participate in the study were recruited.

\section{Exclusion criteria}

The patients were excluded in the study with the criteria such as, patients with previous history of antibiotic resistance, indiscriminate use of antibiotics such as (use of antibiotics without the supervision of registered medical practitioner or violating his/ her direction of use and non-adherent to prescription and use of antibiotics as self medications), immune compromised patients, pyelo-nephritis, known history of drug resistant uropathogens and recurring UTIs, and the patients those who are unwilling to participate are excluded.

\section{Antibiotic susceptibility test}

In vitro antibiotic susceptibility tests on urinary pathogens were performed using the National Committee for Clinical Laboratory Standards' basic agar disc diffusion technique. For the procedure, 11 antibiotics were used, such as Colistin, Amikacin,

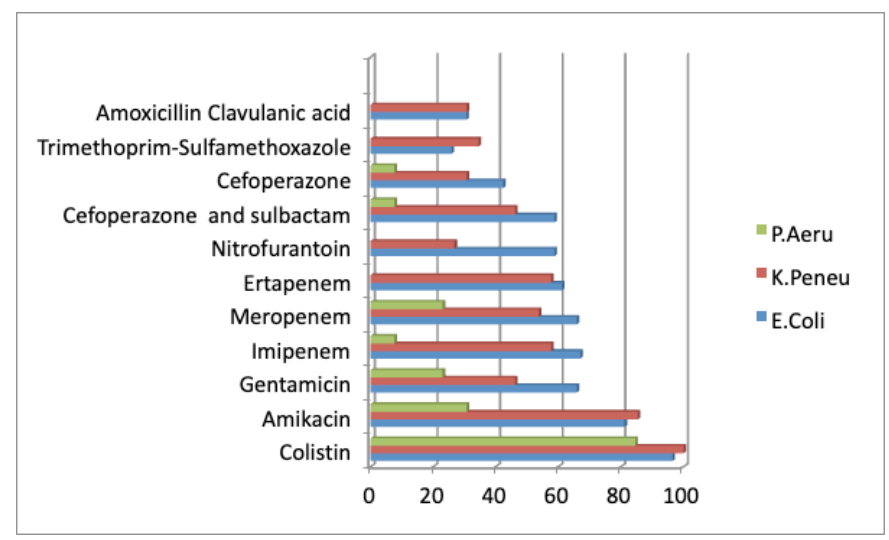

Figure 1. Percentage of antibiotic susceptibility of pathogens in in-patients.

Imipenem, Meropenem, Gentamicin, Ertapenem, Nitrofurantoin, Cefoperazone and sulbactam, Cefoperazone, TrimethoprimeSulfamethoxazole (TMP-SMX), and Amoxicillin Clavulanic acid. An automated (disc diffusion technique) for antimicrobial susceptibility testing systems was used for our study.

\section{Statistical analysis}

For categorical data, summary statistics were calculated using the frequency and proportion. The Chi-square test was applied to compare difference in uropathogen prevalence and resistance trends between outpatient and inpatient urinary isolates. The $p$ value with $<0.05$ was considered to be statistically significant. The Statistical Package for the Social Sciences, IBM and version 21 was used for this study to perform the statistics.

\section{RESULTS}

In our study (Table 1), UTIs are high in in-patients (55.78\%) of males and out-patients females were high $(55.77 \%)$. There was no statistical gender difference among OP and IP patients $(p>0.05)$. Table 2 represents the distribution of Gram negative and Gram positive uropathogens among the in and out patients. A total of 121 E. coli $(60.5 \%), 32(16 \%)$ of $K$. pneumoniae and $18(9 \%)$ were $P$. aeruginosa were observed among the both IP and OP. A 147 IP and 53 OP patients were recruited in our study. Among them, in-patients $85(57.8 \%)$ were isolated with $E$. coli and $36(67.9 \%)$ were observed in out-patients. A very smaller number of Gram positive isolates were observed in our study, and the distribution of isolates are statistically significant among IP and OP patients $(p<0.05)$. Table 3 depicts distribution of Gramnegative uropathogens. A total 181 Gram-negative uropathogens

Table 1. Gender wise distribution of In and Out patients with UTIs.

\begin{tabular}{cccc}
\hline Gender & $\begin{array}{c}\text { In-patients } \\
\boldsymbol{N}=\mathbf{1 4 7}(\mathbf{\%})\end{array}$ & $\begin{array}{c}\text { Out-patients } \\
\boldsymbol{N}=\mathbf{5 3 ( \% )}\end{array}$ & $\boldsymbol{p}$. value \\
\hline Females & $65(44.22)$ & $30(55.77)$ & \\
$N=95$ & & $23(44.23)$ & 0.1232 \\
Males & $82(55.78)$ & & \\
$N=105$ & & & \\
\hline
\end{tabular}

The $p$ value $<0.05$ were considered statistically significant. 


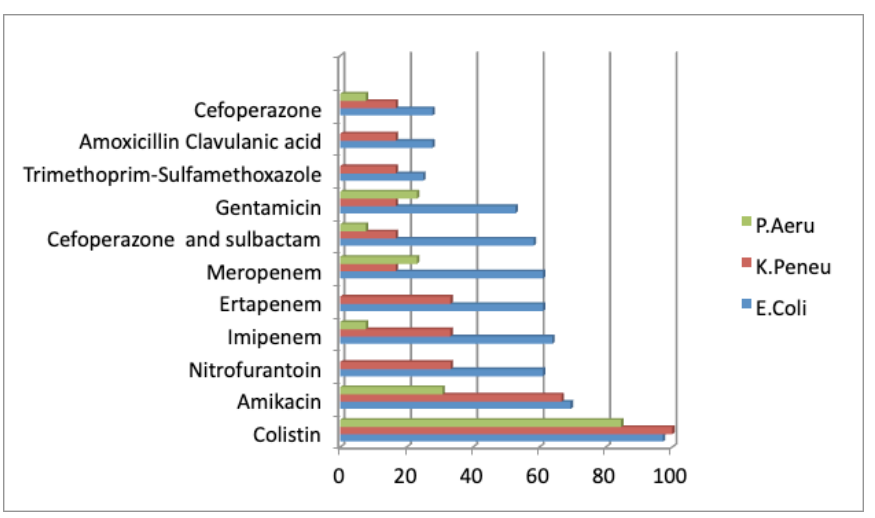

Figure 2. Percentage of antibiotic susceptibility of pathogens in out-patients.

isolates were observed in our study, of which $E$. coli was the most prevalent bacteria among all the age groups. Fifty-eight isolates $(48 \%)$ of $E$. coli were observed in the age range of 60-79 years and $45(35 \%)$ was observed in 40-59 years of age. A 32 of $K$. pneumoniae, 18 of $P$. aeruginosa, and 10 of Staphylococcus aureus were isolated in our study. All Gram-negative bacteria are significant among all the age groups $(p<0.05)$.

Table 4 and Figure 1 indicates the overall antibiotic(s) susceptibility pattern among the different age groups. The antibiotic(s) susceptibility was less than $20 \%$ was noticed in 20 39 years of age groups and less than 10\% were observed in 80-99 years of age groups. The antibiotic susceptibility was found to be $35 \%-40 \%$ among the $40-59$ years of age group, of them Ertapenem and TMP \& SMX only has $48.5 \%$ and $44.6 \%$ of susceptibility. The antibiotic(s) susceptibility was increased $(42 \%-50 \%)$ in 60 79 age groups and toward Ertapenem it was reduced to $26.4 \%$. The antibiotic(s) susceptibility was literally poor in 80-99 years. Table 5 and Figure 2 represents the percentage of susceptibility of antibiotic(s) toward the Gram-negative bacteria. The greatest susceptibility of $E$. coli and $K$. pneumoniae toward Colistin ( $E$. coli-96.7\%, K. pneumoniae-100\%), followed by Amikacin (E. coli-77.7\%, K. pneumoniae-65.6\%), Imipenem (E. coli-66.1\%, K. pneumoniae-53.1\%), and Meropenem (E.coli-64.5\%, K. pneumoniae-46.9\%). A change in susceptibility patterns was seen in case of Pseudomonas aeuriginosa which have shown greater susceptibility to Colistin $(83.3 \%)$, followed by Amikacin (27.8\%), Meropenem (16.7\%), and Gentamicin (16.7\%). TMP and SMZ and Amoxicillin Clavulanic acid were found to be very less susceptibility, i.e., E. coli and $K$. pneumoniae are more resistant $(70 \%)$ with those antibiotics. The pattern of antibiotic (s) susceptibility was not significant among the Gram-negative bacteria $(p>0.05)$.

In-patients, the susceptibility of $E$. coli to Colistin (96.5\%), Amikacin (81.2\%) and $K$. pneumoniae to Colistin (100\%), Amikacin (85.4\%) were observed, respectively. The remaining antibiotics showed $40 \%-67 \%$ of susceptibility. TMP and SMZ and Amoxicillin Clavulanic acid had very poor (less than $30 \%$ ) susceptibility toward the uropathogens (Table 6). In out-patients, Colistin was proved to be very good susceptibility (84.6\%-100\%) toward the Gram-negative bacteria (Table 7). The remaining antibiotics showed less than $70 \%$ of susceptibility toward E. coli and very poor susceptibility toward $K$. pneumoniae $(16 \%-33 \%)$. In contrast to remaining antibiotics such as TMP and SMZ, Amoxicillin Clavulanic acid, Cefoperazone, and Amoxicillin plus Clavulanic acid had relatively low susceptibility $(16 \%-27 \%)$.

\section{DISCUSSION}

Antibiotic resistance is the major challenges in the treatment of UTIs. In our study, we examined the susceptibility of uropathogens toward the antibiotics in IP and OP patients visited with UTIs. The study was conducted during 6 months

Table 2. Distribution of pathogens isolated in urine cultures among in-patients and out-patients.

\begin{tabular}{|c|c|c|c|c|}
\hline Pathogen & $\begin{array}{c}\text { Total } \\
\text { number }(\%)\end{array}$ & $\begin{array}{c}\text { IP } \\
N=147(\%)\end{array}$ & $\begin{array}{c}\text { OP } \\
N=53(\%)\end{array}$ & $p$. value \\
\hline \multicolumn{5}{|l|}{ Gram negative } \\
\hline Escherichia coli & $121(60.5)$ & $85(57.8)$ & $36(67.9)$ & \\
\hline Klebsiella pneumoniae & $32(16)$ & $26(17.7)$ & $6(11.3)$ & \\
\hline Pseudomonas aeruginosa & $18(9)$ & $13(8.8)$ & $5(9.4)$ & \\
\hline Enterococcus & $4(2)$ & $3(2)$ & $1(1.9)$ & \\
\hline Acinetobacter junii & $2(1)$ & $2(1.4)$ & $0(0)$ & $0.007^{*}$ \\
\hline Enterococcus avium & $2(1)$ & $1(0.7)$ & $1(1.9)$ & \\
\hline Enterococcus fecalis & $2(1)$ & $2(1.4)$ & $0(0)$ & \\
\hline \multicolumn{5}{|l|}{ Gram positive } \\
\hline Staphylococcus aureus & $10(5)$ & $8(5.4)$ & $2(3.8)$ & \\
\hline Candida albicans & $5(2.5)$ & $3(2)$ & $2(3.8)$ & \\
\hline Candida & $4(2)$ & $4(2.7)$ & $0(0)$ & \\
\hline
\end{tabular}

\footnotetext{
*The $p$ value $<0.05$ were considered statistically significant.
} 
Table 3. Age Wise Distribution of Gram negative uropathogens.

\begin{tabular}{|c|c|c|c|c|c|c|}
\hline $\begin{array}{c}\text { Age } \\
\text { (Years) }\end{array}$ & $N=181$ & $\begin{array}{l}\text { Escherichia coli } \\
\qquad(N=121 \%)\end{array}$ & $\begin{array}{l}\text { Klebsiella pneumoniae } \\
\qquad(N=\mathbf{3 2} \%)\end{array}$ & $\begin{array}{c}\text { Pseudomonas } \\
\text { aeruginosa } \\
(N=18 \%)\end{array}$ & $\begin{array}{c}\text { Staphylococcus aureus } \\
(N=10 \%)\end{array}$ & $p$ value \\
\hline $20-39$ & 25 & $15(12.4)$ & $5(15.6)$ & $1(5.5)$ & $4(4)$ & \\
\hline $40-59$ & 67 & $45(37)$ & $15(47)$ & $6(33.3)$ & $1(1)$ & \\
\hline $60-79$ & 81 & $58(48)$ & $10(31)$ & $8(44.5)$ & $5(5)$ & $0.03 *$ \\
\hline 80-99 & 8 & $3(2.5)$ & $2(6.3)$ & $3(16.6)$ & $0(0)$ & \\
\hline
\end{tabular}

*The $p$ value $<0.05$ were considered statistically significant.

of period, from 2019 November to 2020 April. There are nearly equal numbers of both genders were recruited in our study. There are more number of in-patients as compared to out-patients with UTI. Out of 200 UTI patients, highest prevalence of bacteria was related to Gram-negative bacteria (E. coli and Klebsiella) (90.5\%) causing UTI and 9.5\% were Gram-positive bacteria. Nitrofurantoin, fosfomycin, sulfanilamides, fluoroquinolones, and beta-lactams are generally recommended antibiotics to treat UTIs (Gupta et al., 2011). The prevalence of isolates was similar in Italian study (Magliano et al., 2011) However, their susceptibility was questionable.

The susceptibility was found to be $35 \%-40 \%$ in $40-59$ years of age group, among them Ertapenem and TMP \& SMX had $48.5 \%$ and $44.6 \%$, respectively. The susceptibility was increased to $42 \%-50 \%$ in $60-79$ years of age group and notably, the low susceptibility (26.4\%) was observed in 60-79 years of age group patients. The antibiotic(s) susceptibility was literally poor in age 80-99; it could be attributed to a weaker immune system, usually seen in this older age group. The results also showed that the highest prevalence of bacteria was related to E. coli in 121 $(60.5 \%)$ and Klebsiella in $32(16 \%)$ of the cases, respectively, and which was consistent with other study (Mohammadi et al., 2010). Among in-patients E. coli, $96.5 \%, 81.2 \%$, and $58.8 \%$ isolates were susceptible to Colistin, Amikacin and Nitrofurantoin, while $K$. pneumoniae, $100 \%, 85.4 \%$, and $26.9 \%$ isolates were susceptible to Colistin, Amikacin, and Nitrofurantoin, respectively. Likewise, in Zambian hospital-based study was nearly correlated with our present study. In Zambia site, in-patients with $K$. pneumoniae and Streptococcus species are resistant to chloramphenicol, Nitrofurantoin, and ceftazidime than out patients (Chanda et al., 2019). In out-patients E. coli, $97.2 \%, 69.4 \%$, and $61.1 \%$ isolates were susceptible to Colistin, Amikacin, and Nitrofurantoin and toward K. pneumoniae it was observed to be $100 \%, 66.7 \%$, and $33.2 \%$. Our findings were quite opposite to the study conducted in Odisha, India, where the E. coli had very low resistant profile toward Amikacin 5.8\% and 9.8\% with Nitrofurantoin (Dash et al., 2013). The similar study conducted in India (Amladi et al., 2019), noticed that, fosfomycin, Nitrofurantoin and Colistin are susceptible to E. coli, $98.9 \%, 56 \%$ and $95 \%$, while fosfomycin and Colistin are susceptible to $94 \%$ and $85 \%$ toward Klebsiella species respectively. Nitrofurantoin and fosfomycin had better susceptibility profile and more effective toward Gram negative bacteria causing uncomplicated UTIs (Gardiner et al., 2019). The similar study conducted in China, reported, ampicillin, cotrimoxazole, ciprofloxacin, amoxicillin, and Nitrofurantoin resistance values in E. coli were $59.8 \%, 31.8 \%, 23.4 \%, 1.9 \%$, and $0.9 \%$ respectively. The results were quite opposite to our study (Wong et al., 2017). Similarly, nalidixic acid and ceftazidime are

Table 4. Age wise distribution of antibiotic susceptibility.

\begin{tabular}{|c|c|c|c|c|c|c|}
\hline \multirow{3}{*}{ Antibiotics } & \multirow{3}{*}{ Number } & \multicolumn{4}{|c|}{ Age in years } & \multirow{3}{*}{$p$ value } \\
\hline & & $20-39$ & $40-59$ & $60-79$ & $80-99$ & \\
\hline & & $N(\%)$ & $N(\%)$ & $N(\%)$ & $N(\%)$ & \\
\hline Colistin & 170 & 19 (11.17) & $65(38.2)$ & $78(45.8)$ & $8(4.7)$ & \\
\hline Amikacin & 124 & $19(15.3)$ & $45(36.2)$ & $55(44.3)$ & $5(4)$ & \\
\hline Imipenem & 98 & $18(18.3)$ & $35(35.3)$ & $42(42.8)$ & $3(3)$ & \\
\hline Meropenem & 84 & $20(23.8)$ & $35(41.67)$ & $27(32.13)$ & $2(2.3)$ & \\
\hline Gentamicin & 94 & $16(17)$ & $35(37.2)$ & $40(42.5)$ & $3(3)$ & $0.142 *$ \\
\hline Ertapenem & 68 & $14(20.5)$ & $33(48.5)$ & $18(26.4)$ & $3(4.4)$ & \\
\hline Nitrofurantoin & 91 & $17(18.6)$ & $26(28.5)$ & $46(50.5)$ & $2(2.1)$ & \\
\hline Cefoperazone and sulbactam & 87 & $18(20.6)$ & $32(36.7)$ & $34(39)$ & $3(3.4)$ & \\
\hline Cefoperazone & 58 & $13(22.4)$ & $22(37.9)$ & $20(34.4)$ & $3(5.1)$ & \\
\hline TMP-SMX & 47 & $6(12)$. & $21(44.6)$ & $18(38.2)$ & $2(4.2)$ & \\
\hline Amoxicillin Clavulanic acid & 45 & $9(20)$ & $17(37.7)$ & $17(37.7)$ & $2(4.4)$ & \\
\hline
\end{tabular}

*The $p$ value $<0.05$ were considered statistically significant. 
Table 5. Percentage susceptibility of uropathogens toward different antibiotics.

\begin{tabular}{|c|c|c|c|c|c|}
\hline \multirow{2}{*}{ Antibiotics } & \multirow{2}{*}{$\%$} & \multicolumn{3}{|c|}{ Organism } & \multirow{2}{*}{$p$ value } \\
\hline & & EC \% & KP \% & PA \% & \\
\hline Colistin & 85 & 96.7 & 100 & 83.3 & \\
\hline Amikacin & 62 & 77.7 & 65.6 & 27.8 & \\
\hline Imipenem & 49 & 66.1 & 53.1 & 5.6 & \\
\hline Meropenem & 48 & 64.5 & 46.9 & 16.7 & 0.58 \\
\hline Gentamicin & 47.5 & 62 & 40.6 & 16.7 & \\
\hline Ertapenem & 45.5 & 61.2 & 53.1 & - & \\
\hline Nitrofurantoin & 45.5 & 59.5 & 28.1 & - & \\
\hline $\begin{array}{l}\text { Cefoperazone } \\
\text { and sulbactam }\end{array}$ & 43.5 & 58.7 & 40.6 & 5.6 & \\
\hline Cefoperazone & 29 & 38 & 28.1 & 5.6 & \\
\hline TMP-SMX & 23.5 & 25.6 & 31.3 & - & \\
\hline $\begin{array}{c}\text { Amoxicillin } \\
\text { Clavulanic acid }\end{array}$ & 22.5 & 29.8 & 28.1 & - & \\
\hline
\end{tabular}

EC: Escherichia coli; KP: Klebsiella pneumonia; PA: Pseudomonas aeruginosa.

more resistant to Enterobacter and Proteus species in females than male patients, in Zambia (Chanda et al., 2019).

In the Iranian settings of both IP and OP departments, the E. coli shows low antibiotic resistant toward Nitrofurantoin (IP$16.5 \%$ and OP-16.3\%), Gentamicin (IP-52.4\% and OP-31.7\%), and ciprofloxacin (IP-60.1\% and OP-34.3\%). High resistant with cotrimoxazole (IP-80\% and OP-62.7\%) and cephalotin (IP-78.3\% and OP-54.3\%) (Davoodabadi et al., 2012). Inpediatrics of IP developed $24 \%$ and $30 \%$ in out-patients resistance toward TMP/

Table 6. Percentage of antibiotic susceptibility of pathogens in in-patients.

\begin{tabular}{cccccc}
\hline Antibiotics & \% & $\mathbf{E C} \%$ & $\mathbf{K P} \%$ & $\mathbf{P A \%}$ & $\boldsymbol{p}$ value \\
\hline Colistin & 84.4 & 96.5 & 100 & 84.6 & \\
Amikacin & 63.9 & 81.2 & 85.4 & 30.8 & \\
Gentamicin & 50.3 & 65.9 & 46.2 & 23.1 & \\
Imipenem & 49.7 & 67.1 & 57.7 & 7.7 & \\
Meropenem & 49.7 & 65.9 & 53.8 & 23.1 & 0.118 \\
Ertapenem & 45.6 & 61.2 & 57.7 & & \\
Nitrofurantoin & 44.2 & 58.8 & 26.9 & & \\
Cefoperazone & 44.2 & 58.8 & 46.2 & 7.7 & \\
and sulbactam & 32 & 42.4 & 30.8 & 7.7 & \\
Cefoperazone & 32.7 & 34.6 & - & \\
TMP-SMX & 24.5 & 25.9 & 30.8 & - & \\
Amoxicillin & 23.1 & 30.6 & 30.6 & \\
Clavulanic acid & & & & & \\
\hline
\end{tabular}

EC: Escherichia coli; KP: Klebsiella pneumonia; PA: Pseudomonas aeruginosa.
SMX with E. coli (Saperston et al., 2014), where in our study we did not have pediatrics. Utility of TMP-SMX was limited in China because of poor sensitivity with E. coli (Zhao et al., 2010). A survey was conducted in Australia; they evaluated the resistance profile of E. coli since 2015-17. They observed that, E. coli had $7 \%$ for ciprofloxacin, $43 \%$ for ampicillin, $9 \%$ for amoxicillin plus clavulanic acid, $16 \%$ for cefazolin, and $22 \%$ for TMP. These findings of TMP were similar to Indian context (Australian Commission on Safety and Quality in Health Care, 2017). In our study, IP E. coli was (IP-67.1\% and OP-63.9\%) K. pneumoniae was (IP-57.7\% and OP-33.2\%) sensitive toward Imipenem. Likewise, in the India, Karala state, the majority of Gram-negative bacteria is susceptible to Imipenem (Thattil et al., 2018). The findings of this study indicate that selecting drugs for empiric treatment would be difficult as no single popular medication can be prescribed for UTI.

\section{CONCLUSION}

Overall, our investigation reports that the resistance of uropathogens to antibiotics was common in both genders of UTI patients. Escherichia coli was more prevalent in 40-79 years of age group. The poor susceptibility was observed in 80-99 years of age groups. Colistin, Amikacin, and Nitrofurantoin showed better susceptibility in in-patients and in out-patients. TMP and SMX, Amoxicillin Clavulanic acid, Cefoperazone, and Amoxicillin plus Clavulanic acid had relatively low susceptibility.

\section{Limitations}

Periodic monitoring of antibiotic susceptibility patterns in clinical settings is vital to determine the potency as well as reestablishing empirical therapy. It is a single center study, hence using this data we cannot generalize the results to whole population. Therefore, the compilation of all the data from different sites are required to establish empirical therapy.

Table 7. Percentage of antibiotic susceptibility of pathogens in out-patients.

\begin{tabular}{cccccc}
\hline Antibiotics & Total & EC & KP & PA & $p$ value \\
\hline Colistin & 85.2 & 97.2 & 100 & 84.6 & \\
Amikacin & 55.6 & 69.4 & 66.7 & 30.8 & \\
Nitrofurantoin & 48.1 & 61.1 & 33.3 & - & \\
Imipenem & 46.3 & 63.9 & 33.2 & 7.7 & \\
Ertapenem & 44.4 & 61.1 & 33.2 & & \\
Meropenem & 42.6 & 61.1 & 16.7 & 23.1 & \\
Cefoperazone and & 40.7 & 58.3 & 16.7 & 7.7 & 0.142 \\
sulbactam & 38.9 & 52.8 & 16.7 & 23.1 & \\
Gentamicin & 20.4 & 25 & 16.7 & - & \\
TMP-SMX & 20.4 & 27.8 & 16.7 & - & \\
Amoxicillin & 20.7 & 16.7 & 7.7 & \\
Clavulanic acid & Cefoperazone & 20.4 & 27.8 & & \\
\hline
\end{tabular}

EC: Escherichia coli; KP: Klebsiella pneumonia; PA: Pseudomonas aeruginosa. 


\section{ACKNOWLEDGMENTS}

The authors are extremely thankful to study participants and the Department of Microbiology, Ramesh Hospital, for their comprehensive assistance and support to execute the research work.

\section{CONFLICT OF INTERESTS}

None.

\section{FUNDING}

Self-funded.

\section{ETHICAL APPROVAL}

The study procedure was approved by the Institutional Ethics Committee, Ramesh Hospitals, (ECR/81/Inst/AP/2019, Dated: 15-10-2019) Vijayawada, India; this research was carried out in conjunction with good clinical practice guidelines.

\section{PUBLISHER'S NOTE}

This journal remains neutral with regard to jurisdictional claims in published institutional affiliation.

\section{REFERENCES}

Akortha EE, Ibadin OK. Incidence and antibiotic susceptibility pattern of Staphylococcus aureus amongst patients with urinary tract infection (UTI) in UBTH Benin City, Nigeria. Afr J Biotechnol, 2008; 7(11):1637-40.

American College of Obstetricians and Gynecologists. ACOG practice bulletin No. 91: treatment of urinary tract infections in nonpregnant women. Obstet Gynecol, 2008; 111(3):785-94.

Amladi AU, Abirami B, Devi SM, Sudarsanam TD, Kandasamy S, Kekre N, Veeraraghavan B, Sahni RD. Susceptibility profile, resistance mechanisms \& efficacy ratios of fosfomycin, nitrofurantoin \& colistin for carbapenem-resistant Enterobacteriaceae causing urinary tract infections. Indian J Med Res, 2019; 149(2):185.

Australian Commission on Safety and Quality in Health Care. AURA 2017: second Australian report on antimicrobial use and resistance in human health. ACSQHC, Sydney, Australia, 2017.

Chanda W, Manyepa M, Chikwanda E, Daka V, Chileshe J, Tembo M, Kasongo J, Chipipa A, Handema R, Mulemena JA. Evaluation of antibiotic susceptibility patterns of pathogens isolated from routine laboratory specimens at Ndola Teaching Hospital: a retrospective study. PLoS One, 2019; 14(12):e0226676.

Dash M, Padhi S, Mohanty I, Panda P, Parida B. Antimicrobial resistance in pathogens causing urinary tract infections in a rural community of Odisha, India. J Fam Commun Med, 2013; 20(1):20.

Davoodabadi A, Farahani A, Ranjbaran M. Antibiotic resistance in Escherichia coli strains isolated from urine of inpatients and outpatients. Zahedan J Res Med Sci, 2012; 14(8):95.

Flores-Mireles AL, Walker JN, Caparon M, Hultgren SJ. Urinary tract infections: epidemiology, mechanisms of infection and treatment options. Nat Rev Microbiol, 2015; 13(5):269-84.

Foxman B. The epidemiology of urinary tract infection. Nat Rey Urol, 2010; 7(12):653-60.

Gardiner BJ, Stewardson AJ, Abbott IJ, Peleg AY. Nitrofurantoin and fosfomycin for resistant urinary tract infections: old drugs for emerging problems. Aust Prescr, 2019; 42(1):14.

Gupta K, Hooton TM, Naber KG, Wullt B, Colgan R, Miller LG, Moran GJ, Nicolle LE, Raz R, Schaeffer AJ, Soper DE. International clinical practice guidelines for the treatment of acute uncomplicated cystitis and pyelonephritis in women: a 2010 update by the infectious diseases society of America and the European society for microbiology and infectious diseases. Clin Infect Dis, 2011; 52(5):e103-20.
Hertz FB, Schønning K, Rasmussen SC, Littauer P, Knudsen JD, Løbner-Olesen A, Frimodt-Møller N. Epidemiological factors associated with ESBL-and non ESBL-producing $E$. coli causing urinary tract infection in general practice. Infect Dis, 2016; 48(3):241-5.

Kidwai SS, Nageen A, Ghaznavi S, Bashir F, Ara J. Antibiotic susceptibility in commonly isolated pathogens from urinary tract infection in a cohort of subjects from low socioeconomic strata. Pak J Med Sci, 2017; 33(2):254.

Langer JL, Chiang KF, Stafford RS. Current prescribing practices and guideline concordance for the treatment of uncomplicated urinary tract infections in women. Am J Obstet Gynecol, 2021; 225:e1-272.

Magliano E, Grazioli V, Deflorio L, Leuci AI, Mattina R, Romano P, Cocuzza CE. Gender and age-dependent etiology of community-acquired urinary tract infections. Sci World J, 2012; 2012:349597.

Mazzulli T. Diagnosis and management of simple and complicated urinary tract infections (UTIs). Can J Urol, 2012; 19(1):42-8.

Medina M, Castillo-Pino E. An introduction to the epidemiology and burden of urinary tract infections. Ther Adv Urol, 2019; 11:1756287219832172.

Mohammadi M, Ghasemi E, Mokhayeri H, Pournia Y, Boroun H. Antimicrobial resistance patterns of $E$. coli detected from hospitalized urine culture samples. Asian J Biol Sci, 2010; 3(4):195-201.

Momoh AR, Orhue PO, Idonije OB, Oaikhena AG, Nwoke EO, Momoh AA. The antibiogram types of Escherichia coli isolated from suspected urinary tract infection samples. J Microbiol Biotech Res, 2011; 1(3):57-65.

Qiao LD, Chen S, Yang Y, Zhang K, Zheng B, Guo HF, Yang B, Niu YJ, Wang Y, Shi BK, Yang WM. Characteristics of urinary tract infection pathogens and their in vitro susceptibility to antimicrobial agents in China: data from a multicenter study. BMJ Open, 2013; 3(12):e004152.

Saperston KN, Shapiro DJ, Hersh AL, Copp HL. A comparison of inpatient versus outpatient resistance patterns of pediatric urinary tract infection. J Urol, 2014; 191(5):1608-13.

Sarwar MI, Sarwar I, Hussain MS, Sherwani SK, Hakeem A, Kazmi SU. Frequency of urinary tract infection causing agents in pregnant women and their antimicrobial susceptibility profile. Pak J Biochem Mol Biol, 2013; 46(3-4):107.

Sharef SW, El-Naggari M, Al-Nabhani D, Al Sawai A, Al Muharrmi Z, Elnour I. Incidence of antibiotics resistance among uropathogens in Omani children presenting with a single episode of urinary tract infection. J Infect Public Health, 2015; 8(5):458-65.

Thattil SJ, Santhosh S. Prevalence of UTI in different age groups in a tertiary care hospital and their antibiogram. Int J Contemp Med Res, 2018; 5(1):3-6.

Wong CK, Kung K, Au-Doung PL, Ip M, Lee N, Fung A, Wong SY. Antibiotic resistance rates and physician antibiotic prescription patterns of uncomplicated urinary tract infections in southern Chinese primary care. PloS One, 2017; 12(5):e0177266.

Zhao L, Chen X, Zhu X, Yang W, Dong L, Xu X, Gao S, Liu X. Prevalence of virulence factors and antimicrobial resistance of uropathogenic Escherichia coli in Jiangsu province (China). Urology, 2009; 74(3):702-7.

\section{How to cite this article:}

Pelluri R, Monika P, Paritala H, Annapareddy CR, Kotha B, Meenavilli S, Angadi SR, Rayapati G, Puttagunta S. Antibiotics susceptibility pattern and prevalence of isolated uropathogens in inpatient and out patients with lower urinary tract infections. J Appl Pharm Sci, 2022; 12(01):159-164. 